\title{
ELASTIC ANALYSIS OF SHEAR WALLS LOADED ANTIMETRICALLY WITH REGARD TO LONGITUDINAL CENTER LINE AND SYMMETRICALLY WITH REGARD TO TRANSVERSAL CENTER LINE PART I
}

\author{
-Airy's Stress Function, Stresses, Displacements, Etc.--
}

\author{
By Dr. MASAHIDE TOMII* and IKUO TOKUHIRO** \\ Members of A.I.J.
}

\section{INTRODUCTION}

In this paper, the formulas for the stresses, strains, and deformations of the symmetrical shearwalls loaded antimetrically with regard to the longitudinal center line and symmetrically with regard: to the transversal center line (see Fig. 4.1) are induced. In general analysis ${ }^{12)}$, these were called. shear wall III subjected to the forces and moments of type III.

The external forces in the $x$-direction are not given arbitrarily, but the eq. 4.1 for the the equilibrium must be satisfied.

$$
\int_{0}^{a}\left(q_{a_{2}}+q_{a e}\right) d x+H_{0}=-\int_{0}^{b} w_{b_{2}} d y
$$

The four corner joints of the boundary frame are assumed as rigid zone (see Fig. 4.1), and thequarter shear wall ( $x \geqq 0, y \geqq 0$ ) is analysed by considering the antimetric loading conditions with. regard to the longitudinal center line and the symmetrical loading conditions with regard to the trans:versal center line.

Dr. Yoshikatsu Tsuboi already analysed the similar shear walls ${ }^{3}$. The differences between Dr. Y. Tsuboi's analysis and this analysis were described in other papers ${ }^{12)}$, and the details of the comparison: will be reported in another paper.

The general analysis was reported in other Transactions ${ }^{122}$ as follows.

$\S 1.1$ Equilibrium for the External Plane Forces and Moments ${ }^{1)}$

$\$ 1.2$ Selection of Serial Stress Functions ${ }^{1}$ )

$\S 1.3$ Elements Not Satisfied only with Serial Stress Function ${ }^{1)}$

$\$ 1.4$ Derivation of the Equations for the Continuity Conditions on the Boundaries between the Wall and Frame ${ }^{1)}$

$\S 1.5$ Equations for Conditions ${ }^{2}$

$\S 1.6$ Selection of Algebraic Stress Functions ${ }^{2)}$

$\S 1.7$ Unit Stresses, Unit Elongations, and Displacements in the Wall ${ }^{2}$

$\$ 1.8$ Stresses, Displacements, etc. in the Upper Beam and Right Column of the Frame ${ }^{2)}$

\section{SYMBOLS}

$H_{0}, V_{0}$ : Horizontal and vertical external forces applied on each corner joint of the frame

$M_{0}:$ External moment applied on each corner joint of the frame

$q_{a_{2}}, q_{b_{2}}$ : External axial shearing force (per unit length) distributed on the outside face of the beam, and that of the column

$w_{a 2}, w_{b_{2}}$ : Sum of the external normal force (per unit length) distributed on the outside face and the

* Prof. of Kyushu Univ., Dr. Eng. ** Assistant of Kyushu Univ. 
external shearing force (per unit length, orthogonal to the axis of the member) distributed on both side faces with regard to the beam, and the similar sum with regard to the column

$q_{a e}, q_{b e}$ : Sum of the external axial shearing forces (per unit length and with the same eccentric distance from the center axis of the member) distributed on both side faces with regard to the beam, and the similar sum with regard to the column

$e_{a}, e_{b}:$ Eccentric distance (from the center axis of the member) of $q_{a e}$, and that of $q_{b e}$

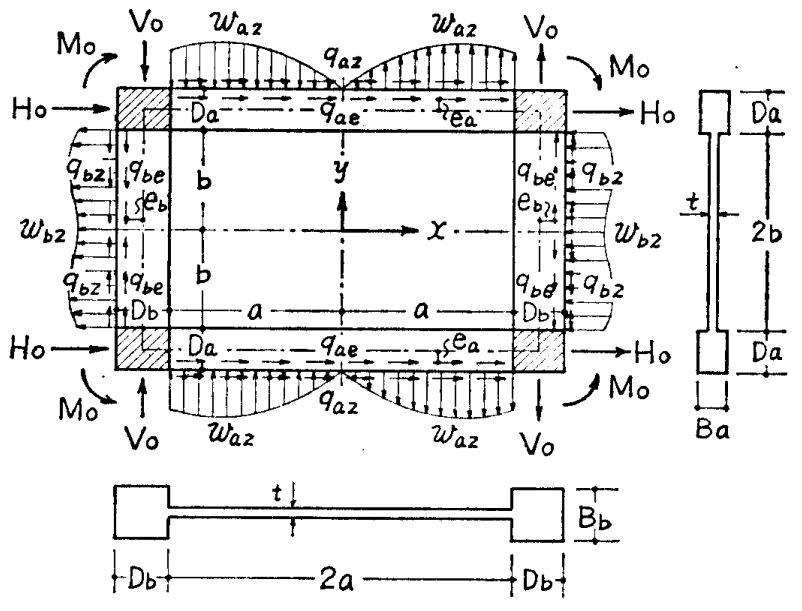

Fig. 4.1 The external forces and moments

$t:$ Thickness of the wall

$D_{a}, D_{b}$ : Depth of the beam, and that of the column

$A_{a}, A_{b}$ : Cross-sectional area of the beam, and that of the column

$I_{a}, I_{b}$ : Geometric moment of inertia of the beam, and that of the column

$Z_{a}, Z_{b}$ : Section modulus of the beam, and that of the column

$E$ : Young's modulus of elasticity

$\nu$ : Poison's ratio

Additional symbols used in this paper are defined where they first appear.

\section{$\$ 4.1$ Airy's Stress Function}

$$
\text { III } F=\sum_{m} F_{s m}+\sum_{n} F_{s n}+{ }_{\text {III }} F_{a}
$$

where,

$$
\begin{aligned}
& { }_{\text {III }} F_{s m}=\left(C_{a m}^{\prime} \cosh \eta_{m}+D_{a m}^{\prime} \eta_{m} \sinh \eta_{m}\right) \sin \xi_{m} \\
& { }_{11} F_{s n}=\left(\begin{array}{llll}
A_{b n}^{\prime} & \sinh \xi_{n}+B_{b n}^{\prime} \quad \xi_{n} \cosh \xi_{n}
\end{array}\right) \cos \eta_{n} \\
& { }_{\text {II }} F_{a}=F_{1} \xi_{a}{ }^{3} \eta_{b}{ }^{2}-\frac{1}{5} \lambda_{b}^{2}\left(F_{1}+\lambda_{b}^{2} F_{2}\right) \xi_{a}^{5}+F_{2} \xi_{a} \eta_{b}{ }^{4}+F_{3} \xi_{a}{ }^{3}+F_{4} \xi_{a} \eta_{b}{ }^{2}
\end{aligned}
$$

${ }_{\text {III } F}:$ Stress function

${ }_{\mathrm{I}} F_{s m},{ }_{\mathrm{II}} F_{s n}$ : Serial stress function (stress function in series)

${ }_{\text {II }} F_{a}:$ Algebraic stress function

$C_{a m}^{\prime}, D_{a m}^{\prime}, A_{b n}^{\prime}, B_{b n}^{\prime}:$ Unknown coefficients in ${ }_{\text {II }} F_{s m}$ and $F_{s n}$

$F_{1}, F_{2}, F_{3}, F_{4}$ : Unknown coefficients in $F_{a}$

$m:$ Odd numbers $1,3,5, \cdots \cdots$

$n$ : Even numbers $2,4,6, \cdots \ldots$

$$
\begin{aligned}
& \xi_{a}=\frac{x}{a}, \quad \xi_{b}=\frac{x}{b}, \quad \xi_{m}=\frac{m \pi}{2} \xi_{a}, \quad \xi_{n}=\frac{n \pi}{2} \xi_{b}, \quad \lambda_{a}=\frac{b}{a}, \quad \alpha_{a m}=\frac{m \pi b}{2 a}, \\
& \eta_{b}=\frac{y}{b}, \quad \eta_{a}=\frac{y}{a}, \quad \eta_{n}=\frac{n \pi}{2} \eta_{b}, \quad \eta_{m}=\frac{m \pi}{2} \eta_{a}, \quad \lambda_{b}=\frac{a}{b}, \quad \alpha_{b n}=\frac{n \pi a}{2 b}
\end{aligned}
$$

\section{$\$ 4.2$ Unit Stresses, Unit Elongations and Displacements in the Wall}

The normal unit stresses $\sigma_{x}$ and $\sigma_{y}$ parallel to $x$ - and $y$-axes respectively and the shearing unit stress $\tau$ in the rectangular coordinates $x$ and $y$ are given as follows.

Here the positive sign should be taken as shown in Fig. 4.2.

$$
\begin{aligned}
\sigma_{y}=\frac{\partial^{2} m F}{\partial x^{2}}= & -\sum_{m}\left(\frac{m \pi}{2 a}\right)^{2}\left(C_{a m}^{\prime} \cosh \eta_{m}+D_{a m}^{\prime} \eta_{m} \sinh \eta_{m}\right) \sin \xi_{m} \\
& +\sum_{n}\left(\frac{n \pi}{2 b}\right)^{2}\left\{\left(A_{b n}^{\prime}+2 B_{b n}^{\prime}\right) \sinh \xi_{n}+B_{b n}^{\prime} \xi_{n} \cosh \xi_{n}\right\} \cos \eta_{n} \\
& +\frac{2}{a^{2}}\left\{3 F_{1} \xi_{a} \eta_{b}{ }^{2}-2 \lambda_{b}{ }^{2}\left(F_{1}+\lambda_{b}{ }^{2} F_{2}\right) \xi_{a}{ }^{3}+3 F_{3} \xi_{a}\right\} \quad \cdots \cdots \cdots(4.3)
\end{aligned}
$$

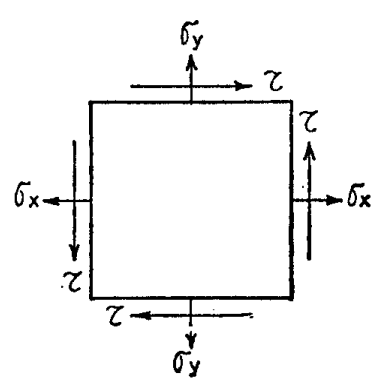

Fig. 4.2 Unit stresses in the wall 


$$
\begin{aligned}
& \left.\sigma_{y}\right|_{y=b}=-\sum_{m}\left(\frac{m \pi}{2 a}\right)^{2}\left(C_{a m}^{\prime} \cosh \alpha_{a m}+D_{a m}^{\prime} \alpha_{a m} \sinh \alpha_{a m}\right) \sin \xi_{m} \\
& +\sum_{n}(-1)^{\frac{n}{2}}\left(\frac{n \pi}{2 b}\right)^{2}\left\{\left(A_{b n}^{\prime}+2 B_{b n}^{\prime}\right) \sinh \xi_{n}+B_{b n}^{\prime} \xi_{n} \cosh \xi_{n}\right\} \\
& +\frac{2}{a^{2}}\left\{3 F_{1} \xi_{a}-2 \lambda_{b}^{2}\left(F_{1}+\lambda_{b}^{2} F_{2}\right) \xi_{a}^{3}+3 F_{3} \xi_{a}\right\} \\
& \sigma_{x}=\frac{\partial^{2}{ }_{\text {III }} F}{\partial y^{2}}=\sum_{m}\left(\frac{m \pi}{2 a}\right)^{2}\left\{\left(C_{a m}^{\prime}+2 D_{a m}^{\prime}\right) \cosh \eta_{m}+D_{a m}^{\prime} \eta_{m} \sinh \eta_{m}\right\} \sin \xi_{m} \\
& -\sum_{n}\left(\frac{n \pi}{2 b}\right)^{2}\left(A_{b n}^{\prime} \sinh \xi_{n}+B_{b n}^{\prime} \xi_{n} \cosh \xi_{n}\right) \cos \eta_{n}+\frac{2}{b^{2}}\left\{F_{1} \xi_{a}{ }^{3}+6 F_{2} \xi_{a} \eta_{b}{ }^{2}+F_{a} \xi_{a}\right) \\
& \left.\sigma_{x}\right|_{x=a}=\sum_{m}(-1)^{\frac{m-1}{2}}\left(\frac{m \pi}{2 a}\right)^{2}\left\{\left(C^{\prime}{ }_{a m}+2 D_{a m}^{\prime}{ }_{a m} \cosh \eta_{m}+D_{a m}^{\prime} \eta_{m} \sinh \eta_{m}\right\}\right. \\
& -\sum_{n}\left(\frac{n \pi}{2 b}\right)^{2}\left(A_{b n}^{\prime} \sinh \alpha_{b n}+B_{b n}^{\prime} \alpha_{b n} \cosh \alpha_{b n}\right) \cos \eta_{n}+\frac{2}{b^{2}}\left(F_{1}+6 F_{2} \eta_{b}^{2}+F_{4}\right) \\
& \tau=-\frac{\partial^{2} \text { II } F}{\partial x \partial y}=-\sum_{m}\left(\frac{m \pi}{2 a}\right)^{2}\left\{\left(C_{a m}^{\prime}+D_{a m}^{\prime}\right) \sinh \eta_{m}+D_{a m}^{\prime} \eta_{m} \cosh \eta_{m}\right\} \cos \xi_{m} \\
& +\sum_{n}\left(\frac{n \pi}{2 b}\right)^{2}\left\{\left(A_{b n}^{\prime}+B_{b n}^{\prime}\right) \cosh \xi_{n}+B_{b n}^{\prime} \xi_{n} \sinh \xi_{n}\right\} \sin \eta_{n}-\frac{2}{a b}\left(3 F_{1} \xi_{a}^{2} \eta_{b}+2 F_{2} \eta_{b}{ }^{3}+F_{4} \eta_{b}\right) \cdots \\
& \left.\tau\right|_{y=b}=\sum_{m} \tau_{m} \cos \xi_{m}-\frac{2}{a b}\left(3 F_{1} \xi_{a}^{2}+2 F_{2}+F_{4}\right)
\end{aligned}
$$

where,

$$
\begin{aligned}
& \tau_{m}=-\left(\frac{m \pi}{2 a}\right)^{2}\left\{\left(C_{a m}^{\prime}+D_{a m}^{\prime}\right) \sinh \alpha_{a m}+D_{a m}^{\prime} \alpha_{a m} \cosh \alpha_{a m}\right\} \\
& \left.\tau\right|_{x=a}=\sum_{n} \tau_{n} \sin \eta_{n}-\frac{2}{a b}\left(3 F_{1} \eta_{b}+2 F_{2} \eta_{b}{ }^{3}+F_{4} \eta_{b}\right) \quad \ldots \ldots \ldots \ldots \ldots \ldots \ldots \ldots \ldots \ldots \ldots \ldots \ldots \ldots
\end{aligned}
$$

where,

$$
\tau_{n}=\left(\frac{n \pi}{2 b}\right)^{2}\left\{\left(A_{b n}^{\prime}+B_{b n}^{\prime}\right) \cosh \alpha_{b n}+B_{b n}^{\prime} \alpha_{b n} \sinh \alpha_{b n}\right\}
$$

The unit elongations $\varepsilon_{x}$ and $\varepsilon_{y}$ parallel to $x$ - and $y$-axes respectively, and the shearing unit strain $r$ are given as follows from eqs. 4.3, 4.5, and 4.7.

Here the positive sign of $\gamma$ should be taken if $\gamma$ is caused by positive $\tau$.

$$
\begin{aligned}
& \varepsilon_{x}=\frac{\partial u}{\partial x}=\frac{1}{E}\left(\sigma_{x}-\nu \sigma_{y}\right)=\frac{1}{E}\left(\sum_{m}\left(\frac{m \pi}{2 a}\right)^{2}\left[C_{a m}^{\prime}(1+\nu) \cosh \eta_{m}+D_{a m}^{\prime}\left\{2 \cosh \eta_{m}+(1+\nu) \eta_{m} \sinh \eta_{m}\right\}\right] \sin \xi_{m}\right. \\
& -\sum_{n}\left(\frac{n \pi}{2 b}\right)^{2}\left[A_{b n}^{\prime}(1+\nu) \sinh \xi_{n}+B_{b n}^{\prime}\left\{2 \nu \sinh \xi_{n}+(1+\nu) \xi_{n} \cosh \xi_{n}\right\}\right] \cos \eta_{n} \\
& \left.+\frac{2}{a^{2}}\left[\lambda_{b}^{2}\left(F_{1} \xi_{a}^{3}+6 F_{2} \xi_{a} \eta_{b}^{2}+F_{4} \xi_{a}\right)-\nu\left\{3 F_{1} \xi_{a} \eta_{b}^{2}-2 \lambda_{b}^{2}\left(F_{1}+\lambda_{b}^{2} F_{2}\right) \xi_{a}^{3}+3 F_{3} \xi_{a}\right\}\right]\right)
\end{aligned}
$$

$$
\left.\varepsilon_{x}\right|_{y=b}=\frac{1}{E}\left(\begin{array}{c}
\sum_{m}\left(\frac{m \pi}{2 a}\right)^{2}\left[C_{a m}^{\prime}(1+\nu) \cosh \alpha_{a m}+D_{a m}^{\prime}\left\{2 \cosh \alpha_{a m}+(1+\nu) \alpha_{a m} \sinh \alpha_{a m}\right\}\right] \sin \xi_{m} \\
\quad-\sum_{n}(-1)^{\frac{n}{2}}\left(\frac{n \pi}{2 b}\right)^{2}\left[A_{b n}^{\prime}(1+\nu) \sinh \xi_{n}+B_{b n}^{\prime}\left\{2 \nu \sinh \xi_{n}+(1+\nu) \xi_{n} \cosh \xi_{n}\right\}\right] \\
\quad+\frac{2}{a^{2}}\left[\lambda_{b}{ }^{2}\left(F_{1} \xi_{a}{ }^{3}+6 F_{2} \xi_{a}+F_{4} \xi_{a}\right)-\nu\left\{3 F_{1} \xi_{a}-2 \lambda_{b}{ }^{2}\left(F_{1}+\lambda_{b}{ }^{2} F_{2}\right) \xi_{a}{ }^{3}+3 F_{3} \xi_{a}\right\}\right]
\end{array}\right)
$$

$$
\varepsilon_{y}=\frac{\partial v}{\partial y}=\frac{1}{E}\left(\sigma_{y}-\nu \sigma_{x}\right)=\frac{1}{E}\left(\begin{array}{l}
-\sum_{m}\left(\frac{m \pi}{2 a}\right)^{2}\left[C_{a m}^{\prime}(1+\nu) \cosh \eta_{m}+D_{a m}^{\prime}\left\{2 \nu \cosh \eta_{m}+(1+\nu) \eta_{m} \sinh \eta_{m}\right\}\right] \sin \xi_{m} \\
+\sum_{n}\left(\frac{n \pi}{2 b}\right)^{2}\left[A_{b n}^{\prime}(1+\nu) \sinh \xi_{n}+B_{b n}^{\prime}\left\{2 \sinh \xi_{n}+(1+\nu) \xi_{n} \cosh \xi_{n}\right\}\right] \cos \eta_{n} \\
+\frac{2}{b^{2}}\left[\lambda_{a}{ }^{2}\left\{3 F_{1} \xi_{a} \eta_{b}{ }^{2}-2 \lambda_{b}{ }^{2}\left(F_{1}+\lambda_{b}{ }^{2} F_{2}\right) \xi_{a}{ }^{3}+3 F_{3} \xi_{a}\right\}-\nu\left(F_{1} \xi_{a}{ }^{3}+6 F_{2} \xi_{a} \eta_{b}{ }^{2}+F_{4} \xi_{a}\right)\right]
\end{array}\right)
$$

$\left.\varepsilon_{y}\right|_{x=a}=\frac{1}{E}\left(-\sum_{m}(-1)^{\frac{m-1}{2}}\left(\frac{m \pi}{2 a}\right)^{2}\left[C^{\prime}{ }_{a m}(1+\nu) \cosh \eta_{m}+D^{\prime}{ }_{a m}\left\{2 \nu \cosh \eta_{m}+(1+\nu) \eta_{m} \sinh \eta_{m}\right\}\right]\right.$ 


$$
\left(\begin{array}{l}
+\sum_{n}\left(\frac{n \pi}{2 b}\right)^{2}\left[A_{b n}^{\prime}(1+\nu) \sinh \alpha_{b n}+B_{b n}^{\prime}\left\{2 \sinh \alpha_{b n}+(1+\nu) \alpha_{b n} \cosh \alpha_{b n}\right\}\right] \cos \eta_{n} \\
+\frac{2}{b^{2}}\left[\lambda_{a}^{2}\left\{3 F_{1} \eta_{b}^{2}-2 \lambda_{b}^{2}\left(F_{1}+\lambda_{b}^{2} F_{2}\right)+3 F_{3}\right\}-\nu\left(F_{1}+6 F_{2} \eta_{b}{ }^{2}+F_{4}\right)\right]
\end{array} \mid\right.
$$

$$
r=\frac{2(1+\nu)}{E} \tau=\frac{2(1+\nu)}{E}\left(\begin{array}{l}
-\sum_{m}\left(\frac{m \pi}{2 a}\right)^{2}\left\{C_{a m}^{\prime} \sinh \eta_{m}+D^{\prime}{ }_{a m}\left(\sinh \eta_{m}+\eta_{m} \cosh \eta_{m}\right)\right\} \cos \xi_{m} \\
+\sum_{n}\left(\frac{n \pi}{2 b}\right)^{2}\left\{A^{\prime}{ }_{b n} \cosh \xi_{n}+B^{\prime}{ }_{b n}\left(\cosh \xi_{n}+\xi_{n} \sinh \xi_{n}\right)\right\} \sin \eta_{n} \\
-\frac{2}{a b}\left(3 F_{1} \xi_{a}{ }^{2} \eta_{b}+2 F_{2} \eta_{b}{ }^{3}+F_{4} \eta_{b}\right)
\end{array}\right)
$$

The displacements $u$ and $v$ parallel to $x$ - and $y$ - axes respectively are given as follows. Here the positive sign should be taken if the $u$ and $v$ have the positive directions of the $x$ - and $y$-axes.

$$
\begin{aligned}
u=\int \frac{\partial u}{\partial x} d x=\frac{1}{E} & -\sum_{m}\left(\frac{m \pi}{2 a}\right)\left[C_{a m}^{\prime}(1+\nu) \cosh \eta_{m}+D_{a m}^{\prime}\left\{2 \cosh \eta_{m}+(1+\nu) \eta_{m} \sinh \eta_{m}\right\}\right] \cos \xi_{m} \\
& -\sum_{n}\left(\frac{n \pi}{2 b}\right)\left[A^{\prime}{ }_{b n}(1+\nu) \cosh \xi_{n}+B_{b n}^{\prime}\left\{-(1-\nu) \cosh \xi_{n}+(1+\nu) \xi_{n} \sinh \xi_{n}\right\}\right] \cos \eta_{n} \\
& \left.+\frac{1}{a}\left[\lambda_{b}{ }^{2}\left(\frac{1}{2} F_{1} \xi_{a}{ }^{4}+6 F_{2} \xi_{a}{ }^{2} \eta_{b}{ }^{2}+F_{4} \xi_{a}{ }^{2}\right)-\nu\left\{3 F_{1} \xi_{a}{ }^{2} \eta_{b}{ }^{2}-\lambda_{b}{ }^{2}\left(F_{1}+\lambda_{b}{ }^{2} F_{2}\right) \xi_{a}{ }^{4}+3 F_{3} \xi_{a}{ }^{2}\right\}\right]\right] \\
& +f_{u}(y)+u_{0}
\end{aligned}
$$

$$
v=\int \frac{\partial v}{\partial y} d y=\frac{1}{E}\left(\begin{array}{l}
-\sum_{m}\left(\frac{m \pi}{2 a}\right)\left[C^{\prime}{ }_{a m}(1+\nu) \sinh \eta_{m}+D^{\prime}{ }_{a m}\left\{-(1-\nu) \sinh \eta_{m}+(1+\nu) \eta_{m} \cosh \eta_{m}\right\}\right] \sin \xi_{m} \\
+\sum_{n}\left(\frac{n \pi}{2 b}\right)\left[A_{b n}^{\prime}(1+\nu) \sinh \xi_{n}+B^{\prime}{ }_{b n}\left\{2 \sinh \xi_{n}+(1+\nu) \xi_{n} \cosh \xi_{n}\right\}\right] \sin \eta_{n} \\
+\frac{2}{b}\left[\lambda_{a}{ }^{2}\left\{F_{1} \xi_{a} \eta_{b}{ }^{3}-2 \lambda_{b}{ }^{2}\left(F_{1}+\lambda_{b}{ }^{2} F_{2}\right) \xi_{a}{ }^{3} \eta_{b}+3 F_{3} \xi_{a} \eta_{b}\right\}\right. \\
\left.-\nu\left(F_{1} \xi_{a}{ }^{3} \eta_{b}+2 F_{2} \xi_{a} \eta_{b}{ }^{3}+F_{4} \xi_{a} \eta_{b}\right)\right]
\end{array}\right)
$$

where, $f_{u}(y)+u_{0}$ is the integration constant in eq. 4.17 , and $f_{\mathfrak{u}}(y)$ which is the even function with regard to $y\left(\eta_{b}\right)$ and the constant $u_{0}$ can be determined as follows.

Note : The integration constant in eq. 4.18 is zero by considering $v_{y=0}=0$ (see eq. 4.57). $\frac{\partial v}{\partial x}=\frac{1}{E}\left(\begin{array}{lll} & -\sum_{m}\left(\frac{m \pi}{2 a}\right)^{2}\left[C^{\prime}{ }_{a m}(1+\nu) \sinh \eta_{m}+D^{\prime}{ }_{a m}\left\{-(1-\nu) \sinh \eta_{m}+(1+\nu) \eta_{m} \cosh \eta_{m}\right\}\right] \cos \xi m \\ & +\sum_{n}\left(\frac{n \pi}{2 b}\right)^{2}\left[A_{b n}^{\prime}(1+\nu) \cosh \xi_{n}+B_{b n}^{\prime}\left\{(3+\nu) \cosh \xi_{n}+(1+\nu) \quad \xi_{n} \sinh \xi_{n}\right\}\right] \sin \eta_{n} \\ & +\frac{2}{a b}\left[\lambda_{a}{ }^{2}\left\{F_{1} \eta_{b}{ }^{3}-6 \lambda_{b}{ }^{2}\left(F_{1}+\lambda_{b}{ }^{2} F_{2}\right) \xi_{a}{ }^{2} \eta_{b}+3 F_{3} \eta_{b}\right\}-\nu\left(3 F_{1} \xi_{a}{ }^{2} \eta_{b}+2 F_{2} \eta_{b}{ }^{3}+F_{4} \eta_{b}\right)\right]\end{array}\right)$

$$
\frac{\partial u}{\partial y}=\frac{1}{E}\left(\begin{array}{l}
-\sum_{m}\left(\frac{m \pi}{2 a}\right)^{2}\left[C_{a m}^{\prime}(1+\nu) \sinh \eta_{m}+D_{a m}^{\prime}\left\{(3+\nu) \sinh \eta_{m}+(1+\nu) r_{m} \cosh \eta_{m}\right\}\right] \cos \xi_{m} \\
+\sum_{n}\left(\frac{n \pi}{2 b}\right)^{2}\left[A_{b n}^{\prime}(1+\nu) \cosh \xi_{n}+B_{b n}^{\prime}\left\{-(1-\nu) \cosh \xi_{n}+(1+\nu) \xi_{n} \sinh \xi_{n}\right\}\right] \sin \eta_{n} \\
+\frac{6}{a b}\left(2 \lambda_{b}{ }^{2} F_{2} \xi_{a}{ }^{2} \eta_{b}-\nu F_{1} \xi_{a}{ }^{2} \eta_{b}\right)
\end{array}\right)+\frac{d f_{u}(y)}{d y}
$$

$$
r=\frac{\partial v}{\partial x}+\frac{\partial u}{\partial y}=\frac{1}{E}\left(\begin{array}{l}
-2(1+\nu) \sum_{m}\left(\frac{m \pi}{2 a}\right)^{2}\left\{C_{a m}^{\prime} \sinh \eta_{m}+D_{a m}^{\prime}\left(\sinh \eta_{m}+\eta_{m} \cosh \eta_{m}\right)\right\} \cos \xi_{m} \\
+2(1+\nu) \sum_{n}\left(\frac{n \pi}{2 b}\right)^{2}\left\{A_{b n}^{\prime} \cosh \xi_{n}+B_{b n}^{\prime}\left(\cosh \xi_{n}+\xi_{n} \sinh \xi_{n}\right)\right\} \sin \eta_{n} \\
+\frac{2}{a b}\left[\lambda_{a}{ }^{2}\left\{F_{1} \eta_{b}{ }^{3}-6 \lambda_{b}{ }^{2}\left(F_{1}+\lambda_{b}{ }^{2} F_{2}\right) \xi_{a}{ }^{2} \eta_{b}+3 F_{3} \eta_{b}\right\}+6 \lambda_{b}{ }^{2} F_{2} \xi_{a}{ }^{2} \eta_{b}\right. \\
\left.-\nu\left(6 F_{1} \xi_{a}{ }^{2} \eta_{b}+2 F_{2} \eta_{b}{ }^{3}+F_{4} \eta_{b}\right)\right]
\end{array}\right.
$$


By making eq. 4.21 equal to eq. 4.16 , eq. 4.22 is obtained.

$$
\frac{d f_{u}(y)}{d y}=-\frac{2}{E a b}\left\{(2+\nu)\left(2 F_{2} \eta_{b}{ }^{3}+F_{4} \eta_{b}\right)+\lambda_{a}{ }^{2}\left(F_{1} \eta_{b}{ }^{3}+3 F_{3} \eta_{b}\right)\right\}
$$

By integrating eq. 4.22 , eq. 4.23 for $f_{\boldsymbol{u}}(y)$ is obtained.

$$
f_{u}(y)=\int \frac{d f_{u}(y)}{d y} d y=-\frac{1}{E a}\left\{(2+\nu)\left(F_{2} \eta_{b}{ }^{4}+F_{4} \gamma_{b}{ }^{2}\right)+\lambda_{a}{ }^{2}\left(\frac{1}{2} F_{1} \eta_{b}{ }^{4}+3 F_{3} \eta_{b}{ }^{2}\right)\right\}
$$

The constant term $u_{0}$ can be obtained from eq. 4.17 by considering $\left.u\right|_{\substack{x=0 \\ y=0}}=0$ (see eq. 4.56).

$$
u_{0}=\frac{1}{E}\left[\sum_{m}\left(\frac{m \pi}{2 a}\right)\left\{C^{\prime}{ }_{a m}(1+\nu)+2 I^{\prime}{ }_{a m}\right\}+\underset{n}{\nu}\left(\frac{n \pi}{2 b}\right)\left\{A_{b n}^{\prime}(1+\nu)-B_{b n}^{\prime}(1-\nu)\right\}\right]
$$

By putting $y=b$ in eq. 4.18 , eq. 4.25 is obtained.

$\therefore v_{y=b}^{\prime}=\sum_{m} v_{m} \sin \xi_{m}+\frac{2}{E b}\left[\lambda_{a}{ }^{2}\left\{F_{1} \xi_{a}-2 \lambda_{b}{ }^{2}\left(F_{1}+\lambda_{b}{ }^{2} F_{2}\right) \xi_{a}{ }^{3}+3 F_{3} \xi_{a}\right\}-\nu\left(F_{1} \xi_{a}{ }^{3}+2 F_{2} \xi_{a}+F_{4} \xi_{a}\right)\right]$

where,

$$
\left.v_{m}=-\frac{1}{E}\left(\frac{m \pi}{2 a}\right)\left[C^{\prime}{ }_{a m}(1+\nu) \sinh \alpha_{a m}+1\right)^{\prime}{ }_{a m}\left\{-(1-\nu) \sinh \alpha_{a m}+(1+\nu) \alpha_{a m} \cosh \alpha_{a m}\right\}\right] \cdots
$$

By substituting eq. 4.23 in eq. 4.17 and putting $x=a$, eq. 4.27 is obtained.

$$
\begin{aligned}
& u_{x: a}=\sum_{n} u_{n} \cos \eta_{n}+\frac{1}{E a}\left[\lambda_{b}{ }^{2}\left(\frac{1}{2} F_{1}+6 F_{2} \gamma_{b}{ }^{2}+F_{4}\right) \cdots \nu\left\{3 F_{1} \eta_{b}{ }^{2}-\lambda_{b}{ }^{2}\left(F_{1}+\lambda_{b}{ }^{2} F_{2}\right)+3 F_{3}\right\}\right. \\
& \left.-(2+\nu)\left(F_{2} \gamma_{b}{ }^{4}+F_{4} \gamma_{b}{ }^{2}\right)-\lambda_{a}{ }^{2}\left(\frac{1}{2} F_{1} \eta_{b}{ }^{4}+3 F_{3} \eta_{b}{ }^{2}\right)\right]+\frac{1}{E}\left[\sum_{m}\left(\frac{m \pi}{2 a}\right)\left\{C^{\prime}{ }_{a m}(1+\nu)+2 D^{\prime}{ }_{a m}\right\}\right. \\
& \left.+\sum_{n}\left(\frac{n \pi}{2 b}\right)\left\{A_{b n}^{\prime}(1+\nu)-B_{b n}^{\prime}(1-\nu)\right\}\right] \\
& u_{n}=\frac{1}{E}\left(\frac{n \pi}{2 b}\right)\left[A_{b n}^{\prime}(1+\nu) \cosh \alpha_{b n}+B_{b n}^{\prime}\left\{\cdots(1-\nu) \cosh \alpha_{b n}+(1+\nu) \alpha_{b n} \sinh \alpha_{b n}\right\}\right] \\
& u_{\substack{x=a \\
y=b}}=\sum_{n}(-1)^{-\frac{n}{2}} u_{n}+\frac{1}{E a}\left[\lambda_{b}{ }^{2}\left(\frac{1}{2} F_{1}+6 F_{2}+F_{4}\right)-\nu\left\{3 F_{1}-\lambda_{b}{ }^{2}\left(F_{1}+\lambda_{b}{ }^{2} F_{2}\right)+3 F_{3}\right\}\right. \\
& \left.\cdots(2+\nu)\left(F_{2}+F_{4}\right)-\lambda_{a}{ }^{2}\left(\frac{1}{2} F_{1}+3 F_{3}\right)\right]+\frac{1}{E}\left[\sum_{m}\left(\frac{m \pi}{2 a}\right)\left\{C^{\prime}{ }_{a m}(1+\nu)+2 I\right)^{\prime}{ }_{a m}\right\} \\
& \left.+\sum_{n}\left(\frac{n \pi}{2 b}\right)\left\{A_{b n}^{\prime}(1+\nu)-B_{b n}^{\prime}(1-\nu)\right\}\right]
\end{aligned}
$$

\section{\$4.3 Stresses, Displacements, etc. in the Upper Beam and Right Column of the Frame}

$\mathrm{B}_{y}$ making the deflection $v_{a}$ of the beam equal to that $v_{y=b}$ of the wall, the relationship (eq. 4.31) between the bending moment and the curvature of the beam is obtained and the other stresses, unit elongations, and displacements of the beam are obtained as follows.

The slope of the beam :

$$
\frac{d v_{a}}{d x}=\left.\frac{\partial v}{\partial x}\right|_{y=b} \sum_{m}\left(\frac{m \pi}{2 a}\right) v_{m} \cos \xi_{m}+\frac{2}{E a b}\left[\lambda_{a}^{2}\left\{F_{1}-6 \lambda_{b}^{2}\left(F_{1}+\lambda_{b}^{2} F_{2}\right) \xi_{a}^{2}+3 F_{3}\right\}-\nu\left(3 F_{1} \xi_{a}^{2}+2 F_{2}+F_{4}\right)\right]
$$

The bending moment of the beam :

$$
\begin{aligned}
M_{a}= & E I_{a} \frac{d^{2} v_{a}}{d x^{2}}=-E I_{a}\left[\sum_{m}\left(\frac{m \pi}{2 a}\right)^{2} v_{m} \sin \xi_{m}+\frac{12}{E a^{2} b}\right. \\
& \left.\cdot\left\{2\left(F_{1}+\lambda_{b}{ }^{2} F_{2}\right) \xi_{a}+\nu F_{1} \xi_{a}\right\}\right] \ldots \ldots \ldots \ldots \cdots \cdots \cdots \cdots \cdots \cdots \cdots \cdots
\end{aligned}
$$

If the external forces (per unit length) on the beam are given in the Fourier series as follows (see Fig. 4.3 a):

$$
\begin{aligned}
& q_{a_{2}}=q_{a_{20} o}+\sum_{m} q_{a_{2 m}} \cos \xi_{m} \\
& q_{a e}=q_{a e o}+\sum_{m} q_{a e m} \cos \xi_{m} \\
& w_{a_{2}}=\sum_{m} w_{a_{2} m} \sin \xi_{m} \ldots \ldots .
\end{aligned}
$$

the stresses, unit elongations, and displacements of the beam can be obtained as follows.

The shearing force on the cross section of the beam :

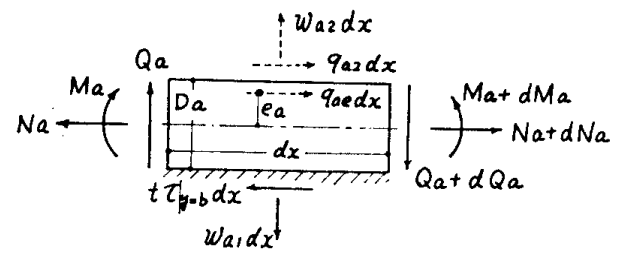

Fig. $4.3 \mathrm{a}$ The forces and moments on the segment $D_{a} \times d x$ of the upper beam

References of Fig. $4.3 \mathrm{a}$ :

i The forces and moments have a positive sign.

ii The forces shown with dotted line are the given external forces per unit length.

iii The hatched part is the in-filled panel wall. 


$$
\begin{aligned}
Q_{a}= & \frac{d M_{a}}{d x}-\frac{D_{a}}{2}\left(\left.t \tau\right|_{y=b}+q_{a_{2}}\right)-e_{a} q_{a e} \\
= & -\sum_{m}\left\{E I_{a}\left(\frac{m \pi}{2 a}\right)^{3} v_{m}+\frac{D_{a}}{2}\left(t \tau_{m}+q_{a 2 m}\right)+e_{a} q_{a e m}\right\} \cos \xi_{m} \\
& -\frac{12 I_{a}}{a^{3} b}\left\{2\left(F_{1}+\lambda_{b}^{2} F_{2}\right)+\nu F_{1}\right\}+\frac{t D_{a}}{a b}\left(3 F_{1} \xi_{a}^{2}+2 F_{2}+F_{4}\right)-\left(\frac{I_{a}}{2} q_{a 2 o}+e_{a} q_{a e o}\right)
\end{aligned}
$$

The normal force (per unit length) distributed on the inside face (boundary adjacent to the wall) of the beam :

$$
\begin{aligned}
w_{a 1} & =-\frac{d Q_{a}}{d x}+w_{a 2} \\
& =-\sum_{m}\left\{E I_{a}\left(\frac{m \pi}{2 a}\right)^{4} v_{m}+\left(\frac{m \pi}{2 a}\right)\left(\frac{t D_{a}}{2} \tau_{m}+\frac{D_{a}}{2} q_{a e m}+e_{a} q_{a e m}\right)-w_{a 2 m}\right\} \sin \xi_{m}-\frac{6 t D_{a}}{a^{2} b} F_{1} \xi_{a}
\end{aligned}
$$

The axial tensile force on the cross section of the beam :

$$
\begin{aligned}
N_{a} & =\left.\int t \tau\right|_{y=b} d x-\int\left(q_{a 2}+q_{a e}\right) d x \\
& =\sum_{m}\left(\frac{2 a}{m \pi}\right)\left(t \tau_{m}-q_{a 2 m}-q_{a e m}\right) \sin \xi_{m}-\frac{2 t}{b}\left(F_{1} \xi_{a}{ }^{3}+2 F_{2} \xi_{a}+F_{4} \xi_{a}\right)-a\left(q_{a 2 o}+q_{a e o}\right) \xi_{a}
\end{aligned}
$$

Note: The integration constant is zero by assuming $\left.N_{a}\right|_{x=0}=0$.

The axial unit elongation on the inside face (boundary adjacent to the wall) of the beam :

$$
\begin{aligned}
\varepsilon_{a 1} & =\frac{1}{E}\left(\frac{M_{a}}{Z_{a}}+\frac{N_{a}}{A_{a}}\right) \\
& =\frac{1}{E}\left(\begin{array}{l}
\sum_{m}\left(\frac{2 a}{m \pi}\right)\left\{-\frac{E I_{a}}{Z_{a}}\left(\frac{m \pi}{2 a}\right)^{3} v_{m}+\frac{t}{A_{a}} \tau_{m}-\frac{1}{A_{a}}\left(q_{a 2 m}+q_{a e m}\right)\right\} \sin \xi_{m} \\
-\frac{12 I_{a}}{Z_{a} a^{2} b}\left\{2\left(F_{1}+\lambda_{b}{ }^{2} F_{2}\right) \xi_{a}+\nu F_{1} \xi_{a}\right\}-\frac{2 t}{A_{a} b}\left(F_{1} \xi_{a}{ }^{3}+2 F_{2} \xi_{a}+F_{1} \xi_{a}\right)-\frac{a}{A_{a}}\left(q_{a 2 o}+q_{a e o}\right) \xi_{a}
\end{array}\right)
\end{aligned}
$$

The axial displacement on the inside face (boundary adjacent to the wall) of the beam :

$$
\begin{aligned}
& u_{a 1}=\int \varepsilon_{a 1} d x=\sum_{m}\left(\frac{2 a}{m \pi}\right)^{2}\left\{\frac{I_{a}}{Z_{a}}\left(\frac{m \pi}{2 a}\right)^{3} \gamma_{m}-\frac{t}{E A_{a}} \tau_{m}+\frac{1}{E A_{a}}\left(q_{a 2 m}+q_{a e m}\right)\right\} \cos \xi_{m} \\
& -\frac{6 I_{a}}{E Z_{a} a b}\left\{2\left(F_{1}+\lambda_{b}{ }^{2} F_{2}\right) \xi_{a}^{2}+\nu F_{1} \xi_{a}^{2}\right\}-\frac{\lambda_{b} t}{E A_{a}}\left(\frac{1}{2} F_{1} \xi_{a}^{4}+2 F_{2} \xi_{a}{ }^{2}+F_{1} \xi_{a}^{2}\right) \\
& -\frac{a^{2}}{2 E A_{a}}\left(q_{a 2 o}+q_{a e o}\right) \tilde{\xi}_{a}^{2}+u_{a 10} \\
& \left.u_{a_{1}}\right|_{x=a}=-\frac{6 I_{a}}{E Z_{a} a b}\left\{2\left(F_{1}+\lambda_{b}^{2} F_{2}\right)+\nu F_{1}\right\}-\frac{\lambda_{b} t}{E A_{a}}\left(\frac{1}{2} F_{1}+2 F_{2}+F_{4}\right)-\frac{a^{2}}{2 E A_{a}}\left(q_{a 2 o}+q_{a e o}\right)+u_{a_{10}}
\end{aligned}
$$

By making $\left.u_{a_{1}}\right|_{x=a}$ equal to the displacement $u_{\substack{x=a \\ y=b}}$ (parallel to $x$-axis, see eq. 4.29) of the corner ( $x=a, y=b$ ) of the wall, the integration constant $u_{a_{10}}$ can be determined as follows.

$$
\begin{aligned}
u_{a 10}= & \sum_{n}(-1)^{\frac{n}{2}} u_{n}+\frac{1}{E a}\left[\lambda_{b}^{2}\left(\frac{1}{2} F_{1}+6 F_{2}+F_{4}\right)-\nu\left\{3 F_{1}-\lambda_{b}{ }^{2}\left(F_{1}+\lambda_{b}^{2} F_{2}\right)+3 F_{3}\right\}-2(2+\nu)\left(F_{2}+F_{4}\right)\right. \\
& \left.-\lambda_{a}^{2}\left(\frac{1}{2} F_{1}+3 F_{3}\right)\right]+\frac{1}{E}\left[\sum_{m}\left(\frac{m \pi}{2 a}\right)\left\{C_{a m}^{\prime}(1+\nu)+2 I^{\prime}{ }_{a m}\right\}+\sum_{n}\left(\frac{n \pi}{2 b}\right)\left\{A_{b n}^{\prime}(1+\nu) \cdots B_{b n}^{\prime}(1-\nu)\right\}\right] \\
& +\frac{6 I_{a}}{E Z_{a} a b}\left\{2\left(F_{1}+\lambda_{b}{ }^{2} F_{2}\right)+\nu F_{1}\right\}+\frac{\lambda_{b} t}{E A_{a}}\left(\frac{1}{2} F_{1}+2 F_{2}+F_{4}\right)+\frac{a^{2}}{2 E A_{a}}\left(q_{a 2 o}+q_{a e o}\right)
\end{aligned}
$$

The axial displacement on the center line of the beam :

$$
\begin{aligned}
& u_{a c}=\int \frac{N_{a}}{E A_{a}} d x=\frac{1}{E A_{a}}\left[\sum_{m}\left(\frac{2 a}{m \pi}\right)^{2}\left(-t \tau_{m}+q_{a 2 m}+q_{a e m}\right) \cos \xi_{m}-\lambda_{b} t\left(\frac{1}{2} F_{1} \xi_{a}^{4}+2 F_{2} \xi_{a}{ }^{2}+F_{4} \xi_{a}{ }^{2}\right)\right. \\
& \left.-\frac{a^{2}}{2}\left(q_{a 2 o}+q_{a e o}\right) \xi_{a}^{2}\right]+u_{a c o}
\end{aligned}
$$




$$
\left.u_{a c}\right|_{x=a}=-\frac{\lambda_{b} t}{E A_{a}}\left(\frac{1}{2} F_{1}+2 F_{2}+F_{4}\right)-\frac{a^{2}}{2 E A_{a}}\left(q_{a 2 o}+q_{a e o}\right)+u_{a c o}
$$

The $u_{a c o}$ is determined from the condition $\left.u_{a c}\right|_{x=a}=\left.u_{a_{1}}\right|_{x=a}-\left.\frac{D_{a}}{2} \frac{d v_{a}}{d x}\right|_{x=a}$, where $u_{a_{1} \mid x=a}$ and $\frac{d v_{a}}{d x}$ are given by eqs. 4.40 and 4.30

$$
\begin{aligned}
u_{a c o}= & \sum_{n}(-1)^{-\frac{u}{2}} u_{n}+\frac{1}{E a}\left[\lambda_{b}^{2}\left(\frac{1}{2} F_{1}+6 F_{2}+F_{4}\right)-\nu\left\{3 F_{1}-\lambda_{b}{ }^{2}\left(F_{1}+\lambda_{b}{ }^{2} F_{2}\right)+3 F_{3}\right\}-2(2+\nu)\left(F_{2}+F_{4}\right)\right. \\
& \left.-\lambda_{a}{ }^{2}\left(\frac{1}{2} F_{1}+3 F_{3}\right)\right]+\frac{1}{E}\left[\sum_{m}\left(\frac{m \pi}{2 a}\right)\left\{C_{a m}^{\prime}{ }_{a m}(1+\nu)+2 D_{a m}^{\prime}\right\}+\sum_{n}\left(\frac{n \pi}{2 b}\right)\left\{A_{a n}^{\prime}(1+\nu)-B_{b n}^{\prime}(1-\nu)\right\}\right] \\
& +\frac{\lambda_{b} t}{E A_{a}}\left(\frac{1}{2} F_{1}+2 F_{2}+F_{4}\right)+\frac{a^{2}}{2 E A_{a}}\left(q_{a 2 o}+q_{a e o}\right)+\frac{\lambda_{a} D_{a}}{E a^{2}}\left\{F_{1}-6 \lambda_{b}{ }^{2}\left(F_{1}+\lambda_{b}{ }^{2} F_{2}\right)+3 F_{3}\right\} \cdots(4.44)
\end{aligned}
$$

By making the deflection $u_{b}$ of the column equal to that $\left.u\right|_{x=a}$ of the wall, the relationship (eq. 4.46) between the bending moment and curvature of the column is obtained and the other stresses, unit elongations, and displacements of the column are obtained as follows.

The slope of the column :

$$
\begin{aligned}
& \frac{d u_{b}}{d y}=\left.\frac{\partial u}{\partial y}\right|_{x=a}=-\sum_{n}\left(\frac{n \pi}{2 b}\right) u_{n} \sin \eta_{n}-\frac{2}{E a b}\left\{3 \nu F_{1} \eta_{b}-6 \lambda_{b}{ }^{2} F_{2} \eta_{b}+\lambda_{a}{ }^{2}\left(F_{1} \eta_{b}{ }^{3}+3 F_{3} \eta_{b}\right)+(2+\nu)\left(2 F_{2} \eta_{b}{ }^{3}\right.\right. \\
& \left.\left.+F_{4} \eta_{b}\right)\right\}
\end{aligned}
$$

The bending moment of the column :

$$
\begin{aligned}
& M_{b}=E I_{b} \frac{d^{2} u_{b}}{d y^{2}}=E I_{b}\left[-\sum_{n}\left(\frac{n \pi}{2 b}\right)^{2} u_{n} \cos \eta_{n}-\frac{2}{E a b^{2}}\left\{3 \nu F_{1}-6 \lambda_{b}{ }^{2} F_{2}+3 \lambda_{a}{ }^{2}\left(F_{1} \eta_{b}{ }^{2}+F_{3}\right)\right.\right. \\
& \left.\left.+(2+\nu)\left(6 F_{2} \eta_{b}{ }^{2}+F_{4}\right)\right\}\right]
\end{aligned}
$$

If the external forces (per unit length) on the column are given in the Fourier series as follows (see Fig. $4.3 \mathrm{~b}$ ) :

$$
\begin{aligned}
& q_{b 2}=\sum_{n} q_{b 2 n} \sin \eta_{n} \ldots \ldots \ldots \ldots \\
& q_{b e}=\sum_{n} q_{b e n} \sin \eta_{n} \ldots \ldots \ldots \ldots \\
& w_{b 2}=w_{b 2 o}+\sum_{n} w_{b 2 n} \cos \eta_{n}
\end{aligned}
$$

the stresses, unit elongtions, and displacements of the beam can be obtained as follows.

The shearing force on the cross sectien of the column :

$$
\begin{aligned}
Q_{b}= & \frac{d M_{b}}{d y}-\frac{D_{b}}{2}\left(\left.t \tau\right|_{x=a}+q_{b 2}\right)-e_{b} q_{b e} \\
= & \sum_{n}\left\{E I_{b}\left(\frac{n \pi}{2 b}\right)^{3} u_{n}-\frac{t D_{b}}{2} \tau_{n}-\left(\frac{D_{b}}{2} q_{b 2 n}+e_{b} q_{b e n}\right)\right\} \sin \eta_{n} \\
& -\frac{12 I_{b}}{a b^{3}}\left\{\lambda_{a}{ }^{2} F_{1} \eta_{b}+2(2+\nu) F_{2} \eta_{b}\right\}+\frac{t D_{b}}{a b}\left(3 F_{1} \eta_{b}+2 F_{2} \eta_{b}{ }^{3}\right. \\
& \left.+F_{4} r_{b}\right) \ldots \ldots \ldots \ldots \ldots \ldots \ldots \ldots \ldots \ldots \ldots \ldots \ldots \ldots \ldots \ldots \ldots \ldots \ldots \ldots \ldots \ldots \ldots \ldots \ldots \ldots \ldots \ldots \ldots \ldots \ldots \ldots \ldots
\end{aligned}
$$

The normal force (per unit length) distributed on the inside face (boundary adjacent to the wall) of the column :

$$
\begin{aligned}
w_{b_{1}}= & -\frac{d Q_{b}}{d y}+w_{b_{2}}=\sum_{n}\left\{-E I_{b}\left(\frac{n \pi}{2 b}\right)^{4} u_{n}+\frac{t D_{b}}{2}\left(\frac{n \pi}{2 b}\right) \tau_{n}\right. \\
& \left.+\left(\frac{n \pi}{2 b}\right)\left(\frac{D_{b}}{2} q_{b_{2} n}+e_{b} q_{b e n}\right)+w_{b 2 n}\right\} \cos \eta_{n} \\
& +\frac{12 I_{b}}{a b^{4}}\left\{\lambda_{a}^{2} F_{1}+2(2+\nu) F_{2}\right\}-\frac{t D_{b}}{a b^{2}}\left(3 F_{1}+6 F_{2} \eta_{b}{ }^{2}+F_{4}\right)+w_{b 2 o}
\end{aligned}
$$

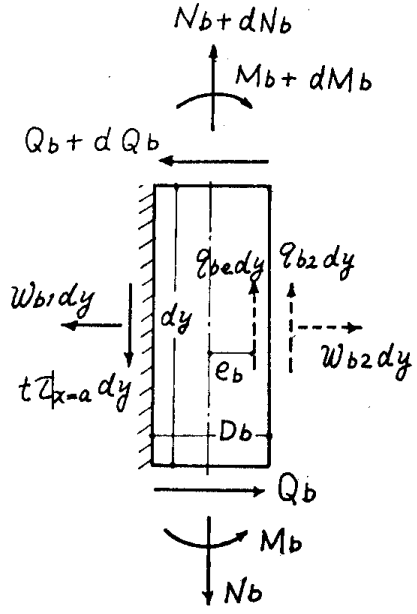

Fig. 4.3 b The forces and moments on the segment $D_{b} \times d y$ of the right column

References of Fig. $4.3 \mathrm{~b}$ :

i The forces and moments have a positive sign.

ii The forces shown with dotted line are the given external forces per unit length.

iii The hatched part is the in-filled panel wall.

The axial tensile force on the cross section of the column :

$$
\begin{aligned}
N_{b} & =\left.t \int \tau\right|_{x=a} d y-\int\left(q_{b 2}+q_{b e}\right) d y \\
& =\sum_{n}\left(\frac{2 a}{n \pi}\right)\left(-t \tau_{n}+q_{b 2 n}+q_{b e n}\right) \cos \eta_{n}-\frac{t}{a}\left(3 F_{1} \eta_{b}{ }^{2}+F_{2} \eta_{b}{ }^{4}+F_{4} \eta_{b}{ }^{2}\right)+N_{b o}
\end{aligned}
$$


where, $N_{b o}$ is the integration constant.

The $N_{b_{0}}$ is the unknown factor in the eq. 4.77 for the continuity of the unit elongations parallel to and on the boundary between the wall and column (see eq. 4.53), and be determined together with many unknown coefficients in the stress function ${ }_{\text {II }} F$ (see eq. 4.2), by solving the simultaneous equations of the conditions mentioned in $\$ 4.4$.

The axial unit elongation on the inside face (boundary adjacent to the wall) of the column:

$$
\begin{aligned}
& \varepsilon_{b_{1}}=\frac{1}{k}\left(\frac{M_{b}}{Z_{b}} \div \frac{N_{b}}{\lambda_{b}}\right) \\
& =\frac{1}{E} \Sigma\left(\frac{2 b}{n \pi}\right)\left\{-\frac{E I_{b}}{Z_{b}}\left(\frac{n \pi}{2 b}\right)^{3} u_{n}-\frac{t}{A_{b}} \tau_{n}+\frac{1}{A_{b}}\left(q_{b 2 n}+q_{b e n}\right)\right\} \cos \eta_{n} \\
& -\frac{2 I_{b}}{Z_{b} a b^{2}}\left\{3 \nu F_{1}-6 \lambda_{b}{ }^{2} F_{2}+(2+\nu)\left(6 F_{2} \eta_{b}{ }^{2}+F_{4}\right)+3 \lambda_{a}{ }^{2}\left(F_{1} \eta_{b}{ }^{2}+F_{3}\right)\right\} \\
& -\frac{1}{\Lambda_{b}}\left\{\frac{t}{a}\left(3 F_{1} r_{b}{ }^{2}+F_{2} \eta_{b}{ }^{4}+F_{4} r_{i}{ }^{2}\right)-N_{b o}\right\}
\end{aligned}
$$

The axial displacement on the inside face (boundary adjacent to the wall) of the column:

$$
\begin{aligned}
& v_{b_{1}}=\int \varepsilon_{b_{1}} d y=\sum_{n}\left(\frac{2 b}{n \pi}\right)^{2}\left\{-\frac{I_{b}}{Z_{b}}\left(\frac{n \pi}{2 b}\right)^{3} u_{n}-\frac{t}{E A_{b}} \tau_{n}+\frac{1}{E A_{b}}\left(q_{b 2 n}+q_{b e n}\right)\right\} \sin \eta_{n} \\
& \frac{2 I_{b}}{E Z_{b} a b}\left\{3 \nu F_{1} \gamma_{b}-6 \lambda_{b}{ }^{2} F_{2} \eta_{b}+(2+\nu)\left(2 F_{2} \eta_{b}{ }^{3}+F_{4} \eta_{b}\right)+\lambda_{a}{ }^{2}\left(F_{1} \eta_{b}{ }^{3}+3 F_{3} \eta_{b}\right)\right\} \\
& -\frac{b}{E A_{b}}\left\{\frac{t}{a}\left(F_{1} r_{b}{ }^{3}+\frac{1}{5} F_{2} \gamma_{b} b^{5}+\frac{1}{3} F_{4} \gamma_{b}{ }^{3}\right)-N_{b o} \gamma_{b}\right\}
\end{aligned}
$$

Note: The integration constant is zero by assuming $\left.v_{b 1}\right|_{y=o}=0$.

The axial displacement on the center line of the column :

$$
\begin{aligned}
\tau^{\prime} b c=\int \frac{N_{b}}{E A_{b}} d y= & \frac{1}{E A_{b}} \sum \frac{2}{n}\left(\frac{2 b}{n \pi}\right)^{2}\left(-t \tau_{n}+q_{b: n}+q_{b e n}\right) \sin \eta_{n} \\
& -\frac{b}{E A_{b}}\left\{\frac{t}{a}\left(F_{1} r_{b}{ }^{3}+\frac{1}{5} F_{2} \gamma_{b}{ }^{5}+\frac{1}{3} F_{4} \gamma_{b}{ }^{3}\right)-N_{b o} \gamma_{i b}\right\}
\end{aligned}
$$

Note: The integration constant is zero by assuming $\left.\tau^{\prime} b c\right|_{y=o}=0$.

\section{NOTE}

As the pages are limited,

$\$ 4.4$ Equations for Condition which are Necessary to Define the Unknown Coefficients and Unknown Factor

$\S 4.5$ Numerical Results of an Example

\section{ACKNOWLEDGEMENT}

\section{REFERENCES}

will be reported in the following 'Transactions as Part II and Part III. 


\section{縦軸に関して逆対称，横軸に関して対称な外力を 受ける耐震壁の弾性解析 第 1 報}

(エアリーの応力関数, 応力度, 变位, そのほか)

\section{序}

本論は，壁板と付帯ラーメンが一体に作られ，䋖横軸 に関してそれぞれ対称な形状および断面を有する鉄筋コ ンクリート造の単独耐震壁に, 綐軸に関し逆対称, 横軸 に関して対称の既知面内外力が付带ラーメンに作用した 場合の平面応力，ひずみ度，変位などの式を誘導したも のである。 $x$ 方向の面内外力は任意に与えることができ ないで，面内外力に関する釣合式 (4.1 式)を満たすよ らに与えなければならない。付带ラーメンの四隅は剛域 とみなし (4.1 図のハッチした部分), 外力が綐軸に関し て逆対称, 横軸に関して対称の条件を考慮して, 耐震壁 の 4 分の 1 の部分 $(x \geqq 0, y \geqq 0)$ につてて解析を行なっ た。

すでに坪井善勝博士は, 縌軸に関し逆対称, 横軸に関 して対称な集中外力が付带ラーメンの節点に作用した耐 震壁の解析を行なっている ${ }^{3)}$ 。坪井博士の解析法と本論 の解析法のちがいはすでに述べたが代,2)，比較の咩細に ついてはさらに追って報告の予定である。

本解析関する一般論は, 次の項目に従ってすでに発 表してある(1,2)ので，それらを参照されたい。

$\S 1.1$ 外力の釣合条件 ${ }^{1)}$

$\S 1.2$ 級数型応力関数の選定 ${ }^{1}$

$\S 1.3$ 級数型応力関数だけでは条件が満足されない要 素1)

$\S 1.4$ 壁板と付帯ラーメンの境界条件式のたて方 ${ }^{12}$

$\S 1.5$ 末知係数および末知積分定数を求めるための条 件式 (連立方程式)

$\S 1.6$ 代数型灾力関数の選定 ${ }^{2)}$

$\S 1.7$ 壁板の応力度, ひずみ度, 変位 ${ }^{23}$

$\S 1.8$ 上ばりおよび右柱の念力, 変形, そのほか ${ }^{21}$

外力，耐震壁の寸法㧍よび断面に関する記号は，4.1 図を参照されたい。本論で使用している諸記号のうちは じめに解説したもの以外のものは, その記号が始めて出 てくる所で説明した。

\section{$\$ 4.1$ エアリーの応力関数}

4.2 式に示すように, エアリーの态力関数として級数

* 九州大学教授・工博 **|i门 助手

(昭和 44 年 6 月 2 日本稿受理・討論期限昭和 45 年 2 月末日)

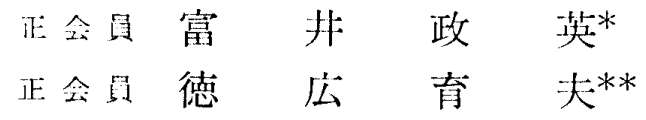

型応力関数と代数型応力関数を併用した。級数型応力閣 数と代数型応力関数を併用して解析を行なら利点や，必 要性などについては，一般論 $\S 1.2^{1)}$ で論じた。

$\$ 4.2$ 壁板の応力度, ひずみ度, 変位

$x y$ ，軸にそれぞれ平行な垂直応力度 $\sigma_{x} \sigma_{y}$, とせん断 忘力度七はそれぞれ 4.5 式, 4.3 式, 4.7 式に示してあ る。符号は 4.2 図に示す方向を正の方向と寸る。

$x, y$ 軸方向の伸縮ひずみ度 $\varepsilon_{x}, \varepsilon_{y}$ およよび $x y$ 軸閒の せん断ひずみ度 $r$ 沬 $\sigma_{y}$ (4.3 式), $\sigma_{x}$ (4.5 式), $\tau(4.7$ 式）から誘導し, それぞれ 4.12 式，4.14 式，4.16 式 に示した。符号は正の応力度によって生じたひずみ度を 正とする。

$x, y$ 軸にそれぞれ平行な変位 $u, v$ はそれぞれ $\varepsilon_{x}, \varepsilon_{y}$ を積分することによって求められる (4.17 式，4.18 式 参照)。 $u$ の積分定数 $f_{u}(y)+u_{0}$ の $5 ち, y\left(\eta_{b}\right)$ の偶関 数で与えられる $f_{u}(y)$ はひずみ度の適合条件から求め たせん断ひずみ度 $r(4.21$ 式) とせん断応力度から剛性 係数を考虑して求めたせん断ひずみ度 $r(4.16$ 式)とを 等しく置くことによって，4.23 式で与えられ，また定数 項 $u_{0}$ は壁板の中心 $(x=0, y=0)$ で $u=0$ という条件 (4.56 式) から 4.24 式で与えられる。vの積分定数は $\left.v\right|_{y=o}=0$ という条件（4.57 式）から零となる。

$\$ 4.3$ 上ばりと右柱の応力, 変位, そのほか 壁板とはりの境界において，はりの垂直変位 $v_{a}$ をつ ーリエ級数と代数関数の和で与えられている壁板とはり の境界上の壁板の垂直変位 $\left.v\right|_{y=b}(4.25$ 式) と等しく置 き, $v_{a}$ を微分してはりの傾斜角を求め (4.30 式), さら に傾斜角を微分して，曲げ剛性を考虑して求められる曲 率と曲げモーメントの関倸から，はりの曲げモーメント を求めると (4.31 式参照)，これらもフーリエ級数と代 数関数の和で表わされる。

はりの軸方向の分布外力 $q_{a 2}, q_{a e}$ および軸に垂直な分 布外力 $w_{a 2}$ (4.3 a 図参照) をそれぞれ 4.32 式, 4.33 式, 4.34 式に示すようなフーリエ級数で与え，これらと 4.8 式で与えられる壁板周辺のせん断忘力度 $\left.\tau\right|_{y=b}$ (注: フー リ工級数と代数関数の和で表わされている）に壁厚 $t$ を 乗じた壁板周辺のせん断㐫力 $\left.t \tau\right|_{y=b}$ をはりに与えると，

1.はりのせん断力 $Q_{a}(4.35$ 式) 
2. はりに分布する垂直分布荷重 $w_{a_{1}}$ (4.36 式)

3. はりの軸方向力 $N_{a}$ (4.37 式)

4. 壁板との境界上のはりの緑ひずみ度 $\varepsilon_{a 1}(4.38$ 式)

5. 壁板との境界上のはりの軸方向変位

$$
u_{a_{1}} \text { (4.39 式) }
$$

注： $u_{a_{1}}$ の積分定数 $u_{a_{10}}$ は，はり端の軸方向変位 $u_{a_{1} \mid x=a}$ を壁板の四隅の $x$ 方向変位 $u_{\substack{x=a \\ y=b}}$ (4. 29 式）と等しく置くことによって求められる (4.41 式参照)。

6. はりの中心線上の軸方向変位 $u_{a c}$ (4.42 式)

注: $u_{a c}$ の積分定数 $u_{a c 0}$ は, $\left.u_{a 1}\right|_{x=a}$ に付帯ラ一 メンの節点 (剛域) の[回転角 (4.30 式に $x=a$ を代入すると得られる) を考虑して求められる (4.44 式参照)。

などもフーリエ級数と代数関数の和で表わされる。

壁板と柱の境界において，柱の垂直変位 $u_{b}$ をフーリ 工級数と代数関数の和で表わされている壁板と柱の境界 上の壁板の垂直変位 $\left.u\right|_{x=a}$ (4.27 式) と等しく置き， $u_{b}$ を微分して柱の傾斜角を求め (4.45 式参照)，さらに傾 斜角を微分して，曲げ岡性を考虑して求められる曲率と 曲げモーメントの関係から，柱の曲げモ一メントを求め ると (4.46 式参照), これらもフーリエ級数と代数関数 の和で表わされる。

柱の軸方向の分布外力 $q_{b 2}, q_{b e}$ および軸に垂直な分 布外力 $w_{b 2}(4.3 \mathrm{~b}$ 図参照) をそれぞれ 4.47 式, 4.48 式, 4.49 式に示すようなフーリエ級数で与えこれらと 4.10 式で与えられる壁板周辺の世ん断応力度 $\left.\tau\right|_{x=a}$ (注： フーリエ級数と代数関数の和で与えられている）に壁厚 $t$ を乗じた壁板周辺のせん断忍力 $\left.t \tau\right|_{x=a}$ を柱に与える と,

1. 柱のせん断力 $Q_{b}$ (4.50 式)

2. 柱に分布する重直分布荷重 $w_{b_{1}}$ (4.51 式)

3. 柱の軸方们力 $N_{b}$ (4.52 式)

注： $N_{b}$ の 積分定数 $N_{b}$ は は, 壁板と柱の境界上の境 界線方问の両者の伸縮ひずみ度の連続条件式 (4.77 式) 中に含まれる未知量であって (4.53 式参照), 忘力関数の未知係数とともに，\$4.4 で述べる各条件式の連立方程式を解くこよによ って求められる。

4. 壁板との境界上の柱の縁ひずみ度 $\varepsilon_{b_{1}}$ (4.53 式)

5. 壁板との境界上の柱の軸方问変位 $v_{b_{1}}$ (4.54 式) 注: $v_{b 1}$ の積分定数は，柱の中央部の軸方向変位が $\left.v_{b_{1}}\right|_{y=0}=0$ であるから零である。

6. 柱の中心線上の軸方向変位. $v_{b c}$ (4.55 式)

注： $v_{b c}$ の積分定数は，柱の中央部の軸方向変位が $\left.v_{b c \mid}\right|_{y=0}=0$ であるから敛である。

などもフーリエ級数と代数関数の和で表わされる。

おことわり

紙再の都含で次の項目は第 2 報および第 3 報として続 報する。

$\S 4.4$ 末知係数および末知積分定数を求めるための条 件式（連立方程式）

$\S 4.5$ 数值针算例

謝 辞

参考文献

注：文中の式および闵は英論文を参照されたい。 